\title{
Artículos
}

\section{Acceso a los recursos web gratuitos desde las bibliotecas'}

\author{
Por Assumpció Estivill y Ernest Abadal
}

\begin{abstract}
Resumen: Se describen y analizan los sistemas que utilizan actualmente las bibliotecas para facilitar a sus usuarios el acceso a los recursos web gratuitos. En primer lugar se acota el ámbito de estudio a este tipo concreto de recursos y se ponen de relieve los principales problemas que tienen los localizadores para su identificación y recuperación. Los modelos que siguen las bibliotecas para organizar los recursos web son básicamente tres: la elaboración de listas, la creación de bases de datos de recursos y la integración de éstos en el catálogo. Este estudio se centra en la descripción, identificación y caracterización de los dos últimos modelos; se destacan las principales experiencias y se comentan los criterios de selección, el tipo de descripción, los sistemas de indización y clasificación, el sistema de recuperación de la información y la política de mantenimiento utilizados en cada uno de ellos. Finalmente, se indican las tendencias actuales en ese ámbito y se presentan unas consideraciones sobre cómo pueden abordar las bibliotecas españolas la organización de estos recursos.
\end{abstract}

Palabras clave: Bibliotecas, Recursos web, Portales temáticos, Catálogos

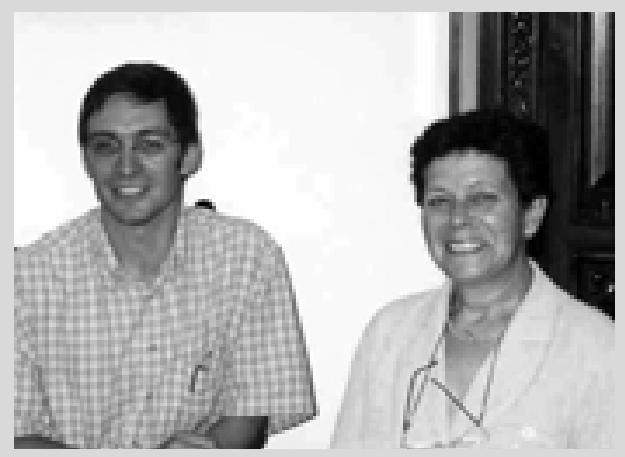

Ernest Abadal y Assumpció Estivill

\section{Title: Access to free web resources from libraries}

Abstract: This article describes and analyses the systems currently used by libraries to provide their users with access to free web resources and it explores the major obstacles for identifying and retrieving these resources. The ways in which libraries organise web resources can be described by three models: making lists, creating databases, and incorporating them into the opac. This study concentrates on the description, identification and characteristics of the latter two approaches, focussing on major experiences with special attention given to the following features in each case: selection criteria, types of descriptions, indexing and classification systems, information retrieval methods, and maintenance policies. Finally, current trends are discussed and considerations are offered as to how Spanish libraries might approach the organisation of web resources.

Keywords: Libraries, Subject gateways, Web resources, Catalogs.

Estivill, Assumpció; Abadal, Ernest. "Acceso a los recursos web gratuitos desde las bibliotecas". En: El profesional de la información, 2000, noviembre, v. 9, $n .11$, pp. 4-20.

Este estudio puede ser útil para los responsables de bibliotecas y centros de documentación españoles que se han planteado qué hacer y cómo tratar las fuentes web que han ido seleccionando y que, de alguna manera, forman parte del fondo documental del propio centro.

Este propósito general tiene una relación directa con el propio origen del estudio, que fue un encargo del Consorci de Biblioteques Universitàries de Catalunya (Cbuc), precisamente para conocer con detalle los modelos y estrategias que existen actualmente en el mundo, y así poder disponer de los elementos de reflexión necesarios para iniciar con más garantías una política de actuación en este ámbito.

\section{Introducción: delimitación del estudio}

La gran cantidad de fuentes de información electrónica disponibles en red, en especial en internet, está teniendo un impacto directo en la definición de las co- lecciones bibliotecarias y en su formación. Actualmente una parte importante de la producción académica o de interés académico (congresos, revistas, informes, proyectos de investigación, etc.) se publica en este medio. Como consecuencia, las colecciones bibliotecarias ya no están formadas exclusivamente por documentos en soportes tangibles que se adquieren por los métodos tradicionales, sino que ahora también pueden formar parte de ellas documentos de acceso remoto que no son necesariamente propiedad de la biblioteca.

Entre los recursos digitales de acceso remoto que pueden tener más repercusiones en el contenido y organización de las colecciones bibliotecarias destacan sobre todo los que se distribuyen en el www. Atendiendo a los procedimientos de adquisición pueden ser de dos tipos: comerciales y gratuitos.

-Entre los comerciales existe todo tipo de documentos y servicios, pero tienen una relevancia especial las bases de datos y las revistas. El sistema de distri-

Recibido el 28-07-00

Aceptación definitiva: 18-09-00 


\begin{tabular}{|c|c|c|c|}
\hline Pais & Nombre & Localización & Especialidad \\
\hline & Anglo-americon culture & 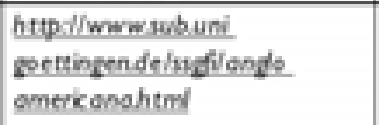 & $\begin{array}{l}\text { Lenga y cultura } \\
\text { angloamericanas }\end{array}$ \\
\hline \multirow[t]{7}{*}{ Alemania } & GesGuide & $\begin{array}{l}\text { http:l/wwwubuni } \\
\text { goettingende/srofigeolind } \\
\text { exheml }\end{array}$ & Geogafia \\
\hline & MathGuide &  & Matemiticas \\
\hline & $\begin{array}{l}\text { Agnic: Agriculture network } \\
\text { informotion center }\end{array}$ & http:-1/wwwognic.org/ & Agricultura \\
\hline & Argos & Hetp:llargosevansilecdu & $\begin{array}{l}\text { Cultura antiga } y \\
\text { medieval }\end{array}$ \\
\hline & CyberStocks (sm) & $\begin{array}{l}\text { httpil/wwe public iostate. } \\
\text { edu/ CYBERSTACXS }\end{array}$ & Multidisoplinar \\
\hline & $\begin{array}{l}\text { Gatewcy to online } \\
\text { resources }\end{array}$ & $\begin{array}{l}\text { http:l/gotewaylbulowas } \\
\text { dulindexhtm }\end{array}$ & Multidisciplinar \\
\hline & $\begin{array}{l}\text { GEM: the goteway to } \\
\text { educational moteriohs }\end{array}$ & http: $/ /$ www geminfo.org/ & \begin{tabular}{|l|}
$\begin{array}{l}\text { Materiales } \\
\text { educitivos }\end{array}$ \\
\end{tabular} \\
\hline \multirow[t]{7}{*}{ Estados Unidos } & Infomine & http-linfomineucredu & Multidisoiplinar \\
\hline & Internet public Bbrory & httpl//werwiplerg & Multidisciplinar \\
\hline & $\begin{array}{l}\text { Lbrovians index to the } \\
\text { internet }\end{array}$ & $\begin{array}{l}\text { http:-1/sungite berkeley odu } \\
\text { Ilnternetindex }\end{array}$ & Multidisoplinar \\
\hline & $\begin{array}{l}\text { Medlineplus: beolth } \\
\text { informetion }\end{array}$ & $\begin{array}{l}\text { httpiliwwerwimnh govim } \\
\text { edlineplus/medlineplushtml/ }\end{array}$ & Medicina \\
\hline & $\begin{array}{l}\text { MEL: The michigan } \\
\text { dectronic Ibrory }\end{array}$ & http:I/melorg & Multidisoiplinar \\
\hline & $\begin{array}{l}\text { Noesa: philosophicel } \\
\text { resterch ondine }\end{array}$ & http:lloseshevansvile edu/ & Filosofia \\
\hline & The scout report signeost & http. $/ /$ scoutcawbedu & Multidisoplinar \\
\hline \multirow[t]{2}{*}{ Francia } & $\begin{array}{l}\text { Camef (Cotologue et } \\
\text { index des sites midicoux } \\
\text { froncophones) }\end{array}$ & $\begin{array}{l}\text { httpiliwerwche } \\
\text { covenffichmefloccuel hem }\end{array}$ & Medicina \\
\hline & Siznets de la Bnf & $\begin{array}{l}\text { httpil/wew bafffr/web } \\
\text { bnfilens/occuel hem }\end{array}$ & Multidisóplinar \\
\hline \multirow[t]{3}{*}{ Paises Bajos } & $\begin{array}{l}\text { DutchESS (Dutch } \\
\text { dectronic subject service) }\end{array}$ & http:l/wwwkenbib. wl/dutchess & Multidisoiplinar \\
\hline & $\begin{array}{l}\text { DEF (Denmark'; dextronic } \\
\text { revearch Bbrary) }\end{array}$ & http:l/wwwdefink.dk/englahl & Multidisciplinar \\
\hline & $\begin{array}{l}\text { Eds (Engheering } \\
\text { dectronk Ibrary, Sweden) }\end{array}$ & http.l/eetshublusel & Ingenieria \\
\hline \multirow[t]{6}{*}{ Paisesnórdicos } & $\begin{array}{l}\text { Finniah virtud Sbrory } \\
\text { project }\end{array}$ & $\begin{array}{l}\text { http:l/wwwijufillbray/vi } \\
\text { rtudolkirjastol }\end{array}$ & Multidisciplinar \\
\hline & Nordic web index & httpiliemiubluse & \\
\hline & $\begin{array}{l}\text { Nerogote: Nordic } \\
\text { gotewoy to information in } \\
\text { forestry, veterindry and } \\
\text { ogricultural aciences }\end{array}$ & $\begin{array}{l}\text { http-illowogote.novo } \\
\text { universityorgl }\end{array}$ & $\begin{array}{l}\text { Agricultura, } \\
\text { veterinaria }\end{array}$ \\
\hline & $\begin{array}{l}\text { Adam (Avt, design, } \\
\text { onchitecture \& media } \\
\text { gotewoy) }\end{array}$ & httpilladomecuk & $\begin{array}{l}\text { Bellas artes, disefio, } \\
\text { artes aplicadas }\end{array}$ \\
\hline & Baz/ed & httpil/wwwbized acesk & Economia y empresa \\
\hline & Bubl & hrtip:libublocuk & Multidisojplimar \\
\hline \multirow[t]{5}{*}{ Reino Unido } & $\begin{array}{l}\text { Eevl (Edinburgh } \\
\text { engineering virtud sbrory) }\end{array}$ & httpdlieevlicbl/hw.oc.uk & Ingenieria \\
\hline & HHR $\sin 60$ & httpdihrsasocuk/ & Historia \\
\hline & $\begin{array}{l}\text { Omil (Organding medical } \\
\text { networked information) }\end{array}$ & http.l/omniocuk & Medicina \\
\hline & $\begin{array}{l}\text { RUDI (Resource for Urban } \\
\text { Design Information) }\end{array}$ & http:linodihertsoc.uk & Urbanismo \\
\hline & $\begin{array}{l}\text { Sorig (Socid science } \\
\text { informetion gotewoy) }\end{array}$ & hrtp:i/sosigacek & Ciendias sodiales \\
\hline
\end{tabular}

Tabla 1. Principales portales temáticos bución de estos productos varía según los casos, ya que pueden estar instalados en un servidor del productor o del distribuidor o, en algunos casos, en uno local administrado por la misma biblioteca ${ }^{2}$. Sus distribuidores ya los dotan de la organización y del sistema de recuperación que facilitarán su explotación y uso. La biblioteca sólo es usuaria del producto y, en la inmensa mayoría de los casos, opta por integrar su descripción en el catálogo (aparte de que pueda elaborar otros instrumentos locales de recuperación).

—Los recursos web gratuitos incluyen documentos y servicios de dominio público de todo tipo (documentos monográficos, bases de datos, catálogos de bibliotecas, revistas, actas de congresos, información corporativa, etc.). Pueden ser de elaboración propia de la organización de la cual forma parte la biblioteca (informes, manuales, tutoriales, etc.), o de producción externa; están en distintos formatos (html, pdf, etc.) y son accesibles para cualquier usuario de la Red. El grado de interés y de calidad de una buena parte de estos recursos justifica que la biblioteca les preste atención y que decida seleccionar los que pueden ser 
útiles para sus usuarios y los organice para ponerlos a su alcance. Este artículo se centra en los sistemas que se han desarrollado para organizar recursos web gratuitos y darles un acceso inmediato.

\section{1. ¿No sirven los localizadores?}

¿Es necesario que las bibliotecas organicen los recursos gratuitos si la misma Red ya dispone de localizadores (directorios temáticos y buscadores) que permiten su identificación y recuperación? Precisamente ha sido el rendimiento poco satisfactorio de estos instrumentos lo que ha motivado el desarrollo de las experiencias que se describen a continuación. Los problemas principales que presentan son los siguientes:

- No disponen de criterios de calidad para seleccionar los recursos que han de formar parte de la base de datos: recuperan todo tipo de documentos de forma indiscriminada.

- Las descripciones de las fuentes indexadas son muy elementales, incompletas y muchas veces erróneas. Normalmente sólo incluyen el título, el URL y en algunas ocasiones un breve resumen o las primeras líneas de la página. Como este proceso es a menudo automático, el número de errores y de omisiones es importante. El usuario puede tener muchas dificultades para decidir los que le interesan cuando el buscador le muestra una lista muy extensa de materiales recuperados a partir de los términos de consulta.

\section{«Las colecciones biblioteca- rias ya no están formadas ex- clusivamente por documentos en soportes tangibles que se adquieren por los métodos tra- dicionales»}

-No presentan todas las opciones de recuperación de los catálogos o de las bases de datos comerciales. Por ejemplo, los usuarios no pueden especificar en qué campo del registro se ha de ejecutar la consulta: una búsqueda por Josep Pla recuperará de manera indiscriminada información sobre este autor, sus propios escritos y documentos publicados por la Fundació Josep Pla.

- La unidad documental de la que parten es el fichero y no el recurso, lo cual provoca un grado muy alto de redundancia.

- La consulta es lenta como consecuencia del tráfico en la Red y de la sobrecarga de algunos servidores.

- Los recursos cambian de localización y los servicios de búsqueda no actualizan sistemáticamente las
URL. No hay, por lo tanto, una política de mantenimiento y actualización de sus bases de datos.

Todas estas deficiencias de los localizadores ofrecen argumentos de peso para diseñar mecanismos alternativos de organización de los recursos web y prefiguran la orientación y las principales características de los sistemas de acceso organizados desde las bibliotecas.

\section{Modelos}

Los escogidos por las bibliotecas para dar acceso a recursos internet seleccionados son básicamente los siguientes:

-Guías de recursos: listas organizadas alfabética o temáticamente. Sólo permiten un acceso secuencial a la información (ya sea por su título, por la materia de que trata o por el tipo de documento). Su elaboración se basa en la selección de las fuentes que incluyen; ahora bien, los criterios para llevar a cabo esta selección no son siempre explícitos. Así mismo, la descripción es muy elemental y poco normalizada y no explotan ningún mecanismo de recuperación de la información.

- Bases de datos de recursos web: las referencias se incluyen en una base de datos separada del catálogo y se gestionan a través de software que facilita su recuperación.

- Recursos web integrados en el catálogo: esta opción exige el mismo tratamiento detallado y normalizado que se realiza con todos los documentos de la colección. Puede ser una alternativa complementaria de la anterior y, de hecho, son diversas las bibliotecas (sobre todo en el entorno norteamericano) que utilizan la referencia Marc como registro básico y único a partir del cual se alimenta el catálogo y se elaboran guías y bases de datos de ciertos tipos de recursos electrónicos de acceso remoto.

Del análisis que se presenta a continuación se ha descartado el primero de los tres modelos, ya que un simple listado alfabético o temático sólo sería aceptable para recopilaciones muy breves y, vistas las dimensiones de internet, esta solución sólo puede ser válida en casos muy concretos y aun de forma provisional. De los otros dos sistemas se enumeran algunas de las experiencias más destacadas y se explican sus características con relación a los aspectos siguientes:

—criterios de selección de los recursos;

—descripción, indización y clasificación;

— sistema de recuperación de la información; y

-mantenimiento. 
De hecho, tanto la formación de bases de datos separadas del catálogo como la integración en éste de los recursos web comparten diversos aspectos. Así por ejemplo, cualquier organización que planifique un procedimiento de acceso a estas fuentes ha de establecer, en primer lugar, los criterios para seleccionarlas y el método de mantenimiento de las direcciones electrónicas. Otras cuestiones (como la descripción de los recursos, el análisis del contenido y el sistema de recuperación de la información) diferirán en mayor o menor medida en función del modelo adoptado para acceder a ellos.

Hasta el momento la mayoría de bibliotecas españolas que ofrecen a sus usuarios recursos web seleccionados han optado por la elaboración de guías. No existen prácticamente experiencias de creación de bases de datos de este tipo ni de su integración en el catálogo. Algunas excepciones que se podrían citar como ejemplo son las bases de datos siguientes:

-Ep!: webs d'interès politècnic de la Universitat Politècnica de Catalunya (Beumala et al., 1998) http://www.upc.es/catala/biblio/biblio.htm

-DinaWEB de la Universitat Oberta de Catalunya que incluye tanto recursos de producción propia como externos (Serrano et al., 1999); y

-Proyecto Darwin (Directorio analítico de recursos web informativos), iniciado en 1998 en el marco del trabajo docente de profesores de bibliografía de diversas universidades españolas (Merlo et al., 1999).

$\mathrm{La}$ inexistencia de experiencias explica que no aparezcan referencias procedentes del entorno hispano en el cuerpo central del texto que ahora presentamos.

\section{Creación de bases de datos de recursos web}

Subject gateways, information gateways, subjectbased information gateways o resource discovery systems (Roszkowski, 1998) son términos que se han consolidado para hacer referencia a las bases de datos que contienen descripciones de recursos web. El proyecto Desire define estas pasarelas o portales temáticos de la forma siguiente: "sitios o servicios en línea que proporcionan catálogos de recursos internet que facilitan su consulta y búsqueda. En general se centran en un conjunto relacionado de áreas temáticas de interés académico (Subject gateways, 1999)".

Las bases de datos de recursos web comparten una serie de características: están especializadas en un área temática determinada ${ }^{3}$; facilitan el acceso a partir de la materia del recurso (de ahí el nombre de subject gateway); tienen una política muy bien definida para el de- sarrollo de la colección y aplican criterios de calidad estrictos; la selección y la descripción de los recursos están a cargo de profesionales y expertos en el campo, lo cual asegura la calidad de la base de datos, y disponen de procedimientos muy claros para el mantenimiento y la actualización de la colección. Por el momento estos portales temáticos tienen carácter gratuito ${ }^{4}$.

El número de servicios que reúnen estas características está aumentando rápidamente. En un trabajo reciente centrado en la descripción de portales temáticos John Kirriemuir (1999) hace referencia a 141 bases de datos de este tipo (64 si se aplican criterios más restrictivos). La mayoría ha sido desarrollada en el Reino Unido y en los Estados Unidos, pero también las hay en los países nórdicos, Holanda, Alemania, Australia, etc. ${ }^{5}$.

El Reino Unido es un país pionero en experiencias de creación de estas herramientas las cuales presentan un grado elevado de desarrollo y coordinación. En general los portales temáticos británicos han sido creados y son actualizados por bibliotecas universitarias y especializadas, estando dirigidos a la comunidad académica tanto docente como investigadora. Una gran parte de estas experiencias ha sido patrocinada por el programa Electronic libraries (eLib) que se puso en marcha en 1995 y que hasta este momento ha subvencionado más de setenta proyectos ${ }^{6}$.

\section{«Las deficiencias de los busca- dores ofrecen argumentos de peso para diseñar mecanismos alternativos de organización de los recursos web y prefiguran la orientación y las principales ca- racterísticas de los sistemas de acceso organizados desde las bibliotecas»}

También en el Reino Unido encontramos la $R e$ source discovery network $(R D N)$, una red cooperativa que agrupa bases de datos temáticas creadas por bibliotecas universitarias e instituciones académicas y de investigación ${ }^{7}$. Su finalidad es facilitar la consulta simultánea de diversas bases de datos. En la actualidad ya funciona como portal que da acceso a diferentes fuentes temáticas de recursos web, algunas de ellas resultado de proyectos cooperativos: Biome (salud y ciencias de la vida), EMC (ingeniería, matemática e informática), Humbul (humanidades), PSIgate (física, química, ciencias de la tierra, historia de la ciencia, medio ambiente, etc.), Sosig (ciencias sociales, derecho y economía). 
En el ámbito europeo destaca el proyecto Desire, subvencionado por la Unión Europea dentro del Programa de aplicaciones telemáticas, que actualmente está ya en su segunda fase $(1998-2000)^{8}$. Su objetivo es promover y facilitar el uso del www entre la comunidad académica europea. Con un enfoque más internacional IMesh ${ }^{9}$ —con sede en la Ukoln (UK Office for Library and Information Networking, University of Bath) - comparte con Desire el desarrollo de tecnologías e instrumentos que mejoren los servicios a los usuarios de recursos internet. También pretende impulsar la cooperación que evite duplicidad de esfuerzos, compartir costes de desarrollo y de software, facilitar la interoperabilidad entre bases de datos, etc.

\section{«Un simple listado alfabético o temático sólo sería aceptable para recopilaciones muy breves $y$, vistas las dimensiones de in- ternet, esta solución sólo puede ser válida en casos muy concre- tos, y aun de forma provisional»}

Dentro de esta misma línea también se puede mencionar el proyecto Renardus ${ }^{10}$, iniciado en enero de 2000, financiado por el programa Tecnologías para la sociedad de la información (IST) de la Unión Europea y dirigido por la Biblioteca Nacional de los Países Bajos. Su objetivo es mejorar el acceso a los recursos web culturales y científicos de tipo académico estableciendo un servicio europeo de subject gateways. Renardus quiere aprovechar el éxito de estos portales temáticos y propone la creación de un modelo de funcionamiento que permita la realización de búsquedas conjuntas por medio de una interfaz única, una especie de catálogo colectivo, cuya estructura aún no se sabe si será de tipo centralizado o distribuido.

Los portales temáticos de Estados Unidos no tienen ni la apariencia homogénea ni el grado de coordinación que presentan los del Reino Unido y de otros países europeos. A diferencia de estos últimos que, a menudo son el resultado de proyectos cooperativos o impulsados desde las bibliotecas nacionales, las numerosas pasarelas temáticas norteamericanas parten, en la mayoría de los casos, de iniciativas de bibliotecas individuales o de consorcios. Entre las excepciones a esta tendencia hay que destacar el proyecto cooperativo Agnic, fomentado por la National Agricultural Library, y Medlineplus de la National Library of Medicine. Así mismo, la Isaac Network, parte del Internet scout project y con subvenciones de la National Science Foundation, tiene el objetivo de investigar la posibilidad de enlazar colecciones de metadatos para formar una colección virtual que permita una consulta unificada. Ha participado en proyectos internacionales y ha colaborado con la citada Ukoln y el Institute for Learning and Research Technology (Ilrt), de la University of Bristol, en el desarrollo del proyecto IMesh Toolkit - conjunto de software con una arquitectura que facilita la interoperabilidad entre distintos portales temáticos (Roszkowski, 1998)—.

Australia también dispone de servicios de estas características. Desde la sede web de la National Library of Australia se da acceso a Australian subject gateways to internet resources ${ }^{11}$ que incluye tanto guías como bases de datos que recopilan recursos web australianos o de temática australiana. En la tabla 1 se presenta una muestra reducida, agrupada por países, de bases de datos temáticas.

\section{Integración de recursos web en el catálogo}

Ésta es una alternativa que algunas bibliotecas practican desde hace tiempo. Desde esta perspectiva, el catálogo se convierte en el portal de acceso a todos los materiales que el centro selecciona para prestar servicios a su comunidad de usuarios, tanto si son de su propiedad, como si son documentos de los que sólo posee licencia de uso y que residen en servidores externos o si son recursos gratuitos ubicados en cualquier servidor de la Red. Algunos autores (Gorman, 1997; Taylor, 1996) dan a esta opción su apoyo incondicional o destacan sus ventajas, que podrían resumirse de la manera siguiente:

-Asegura que los recursos electrónicos sean seleccionados a partir de criterios paralelos a los que se establecen para el resto de materiales de colección y que, por lo tanto, estén conformes con la política global que rige su desarrollo ${ }^{12}$.

- A partir de un instrumento único, el catálogo, el usuario encuentra todos los documentos que la biblioteca ha considerado adecuados para su comunidad y procesados según un esquema uniforme y normalizado.

-Desde el punto de vista de su procesamiento, si bien elaborar registros Marc completos es una tarea costosa, el uso de medios normalizados facilita que puedan ser compartidos e intercambiados.

-A partir del catálogo cualquier documento individual se relaciona con el resto de la colección.

A pesar de las ventajas anteriores no es la opción predominante para todo tipo de recursos internet. El procesamiento y control bibliográfico de estos documentos está en continua evolución.

Actualmente las bibliotecas ya integran en el catálogo la descripción de las bases de datos y de las re- 
vistas digitales comerciales, pero continúan elaborando páginas web con listas de estos documentos ordenados por el título u organizados temáticamente ${ }^{13}$. Éstas, que sirven para mostrar a los usuarios los materiales a los que pueden acceder pero que no ocupan un lugar físico en los estantes, tienen el inconveniente de que obligan al mantenimiento doble de las direcciones electrónicas de los recursos. Para solventar este problema se han desarrollado nuevos modelos que parten de la idea de que el registro Marc del catálogo sea la única localización que sirva para almacenar y mantener las direcciones electrónicas. Knudson (1997) explica la puesta en marcha de uno de estos sistemas en la Research library de Los Alamos National Laboratory ${ }^{14}$.

En el caso de los recursos web gratuitos la tendencia de integrarlos en el catálogo no está tan generalizada. Las bibliotecas han optado más, hasta el momento, por alguno de los otros dos modelos: la elaboración de listas de recursos o la formación de bases de datos específicas. Sin embargo desde hace tiempo hay proyectos y prácticas en este sentido que recientemente están adquiriendo más importancia..

La primera experiencia formal (ya en 1993) de catalogación de recursos electrónicos de acceso remoto con vista a su integración en catálogos de biblioteca fue el Internet resource project de Oclc. Con un enfoque experimental, tenía el objetivo de investigar los problemas teóricos y prácticos de aplicación de la segunda edición revisada de las Aacr2 (Anglo-American cataloging rules) y del formato Marc a aquellos materiales. El estudio contó con la colaboración de 30 profesionales que catalogaron 300 ficheros internet (cada uno realizó 10). El proyecto fue decisivo para el desarrollo del campo 856 de localización y acceso de recursos electrónicos remotos del formato Usmarc.

Oclc ha sido también responsable de tres proyectos más que ya no han sido experimentales como el primero, sino que han tenido un propósito y unos resultados prácticos. En los tres casos se han creado catálogos de recursos internet y, en mayor o menor medida, se han aplicado los instrumentos catalográficos tradicionales -Aacr2, Lcsh (Library of Congress subject headings), Marc, etc.- . Sólo uno de los proyectos, NetFirst, ha sido desarrollado por personal de Oclc; los otros dos, Corc e Internet cataloging project, son o han sido el resultado de la participación voluntaria de numerosas bibliotecas mayoritariamente de los Estados Unidos pero también de otros países.

El Internet cataloging project se inició en 1995 con el propósito de estimular la catalogación de estos materiales y elaborar una base de datos de recursos electrónicos (InterCAT) que posteriormente había de integrarse en el catálogo colectivo WorldCat ${ }^{15}$. Si bien concluyó en 1996, muchas bibliotecas continuaron alimentándolo, y llegó a incluir unos 90.000 registros de todo tipo de recursos web (directorios, buscadores, textos electrónicos, revistas, servicios de información universitarios, etc.) aportados por más de 1.000 bibliotecas ${ }^{16}$. InterCAT demostró que los estándares catalográficos podían aplicarse en la catalogación de materiales internet y ayudó a detectar aquellos aspectos de las normas y de los formatos que requerían revisiones.

NetFirst es otra de las iniciativas de Oclc. Esta base de datos, que incluye también una gran variedad de recursos internet, se inauguró en febrero de 1996 con 40.000 registros. Actualmente contiene más de 100.000 y su crecimiento mensual es de entre 10.000 y 15.000 nuevas aportaciones. Las referencias de cada recurso incluyen su descripción bibliográfica completa, un resumen, los encabezamientos de materia según Lcsh, la clasificación según la Dewey decimal classification $(D D C)$ y el URL. Elaborados con un formato local, los registros pueden convertirse fácilmente al formato Marc desde agosto de $1999^{17}$. NetFirst se caracteriza por su selección cuidadosa, por su calidad (los registros son elaborados con instrumentos normalizados), por la verificación y actualización constante de sus direcciones electrónicas y por la combinación de procedimientos automáticos y manuales para desarrollarla. A diferencia de los otros proyectos de Oclc, que se nutren de registros elaborados por las bibliotecas colaboradoras, NetFirst siempre ha sido alimentado por personal de Oclc.

$\operatorname{Corc}^{18}$ es el proyecto más reciente de $O c l c$ y se basa en la experiencia de los dos anteriores. Su fase de desarrollo se inició en agosto de 1998; entre enero de 1999 y julio de 2000 ha funcionado de forma experimental y ha estado abierto de manera gratuita a todas las bibliotecas que han querido colaborar - unas 480 , la mayoría de los Estados Unidos pero también con una amplia representación de otros países ${ }^{19}$ - . Desde julio de 2000 está disponible como un servicio de pago para los miembros de Oclc.

\section{«Los portales temáticos británi- cos han sido creados y son ac- tualizados por bibliotecas uni- versitarias y especializadas y van dirigidos a la comunidad académica»}

Su objetivo ha sido investigar la creación de un catálogo de recursos internet en el que puedan convivir registros Marc con otros con metadatos menos forma- 
les. En concreto el proyecto parte del conjunto de datos del Dublin core $(D C)$ - ver más adelante- y de los estándares RDF (Resource description framework) y xml (extensible markup language) que son fundamentales para el intercambio de metadatos en el web. Corc facilita también la generación dinámica de páginas de recursos web que puedan integrarse en portales temáticos (pathfinders) ${ }^{20}$. Este aspecto es el más innovador, ya que a partir de un único registro se puede alimentar el catálogo Corc, los catálogos locales de bibliotecas y, al mismo tiempo, generar guías de recursos internet.

La base de datos inicial se formó con registros de InterCAT y de NetFirst, y las bibliotecas participantes han podido crear, editar, importar y exportarlos tanto en Marc como $D C$ (etiquetado en html o en $R D F$ ). Además del catálogo de recursos web, Corc pone a disposición de sus usuarios una serie de bases de datos adicionales que facilitan su catalogación y el uso de instrumentos normalizados. Desde Corc se puede acceder a tesauros como $\mathrm{MeSH}$ y a las siguientes bases de datos: Lcsh, Lcnaf (Library of Congress name authority file), $D D C$ y guías temáticas o pathfinders. Oclc, como parte del proyecto Corc o de otros proyectos de investigación, ha desarrollado software que automatiza la creación de registros, la asignación de clases y subclases de la $D D C$, el control de autoridades, la asignación de palabras clave, el mantenimiento asistido de las direcciones electrónicas de los recursos, y la creación y edición de sus guías digitales. También dispone de un harvester (compilador e indexador de recursos) parametrizable que facilita la localización e identificación de materiales a catalogar (Hickey, 1999). A lo largo de la fase piloto se ha prestado más atención a la catalogación de servicios web y colecciones de materiales que a la de páginas html individuales.

Éste es probablemente el proyecto más completo y complejo de los existentes. Su aspecto más innovador es que representa la integración de los dos modelos examinados: los registros de recursos internet de Corc tienen el doble objetivo de alimentar catálogos y guías o bases de datos de este tipo de fuentes. A través de él se refuerza la idea de que las dos opciones no son excluyentes.

\section{Características de los dos modelos}

En este apartado se describen y comparan los dos sistemas (creación de bases de datos de recursos web e integración de la descripción de éstos en el catálogo) en relación con los aspectos siguientes: criterios de selección; descripción; indización y clasificación; métodos de recuperación de la información y mantenimiento.

\section{Selección}

Las bases de datos de webs, dirigidas muchas de ellas a la comunidad académica, dan prioridad a la categoría de los contenidos y, como consecuencia, son muy selectivas. La mayoría de las citadas anteriormente disponen de documentos formales y públicos en los que se establecen criterios de calidad muy estrictos que rigen la inclusión de recursos en las bases respectivas. Omni, Sosig, Biz/ed, Dutchess, Eels y The scout report, ya citadas, han elaborado documentos de este tipo; entre todos destaca por su exhaustividad y sistematización el documento redactado para Desire (Hofman; Worsfold, 1999). Es precisamente por estos requerimientos cualitativos por lo que el número de registros de estas bases de datos no acostumbra a ser muy elevado. Así por ejemplo, en julio de 2000 había unos 2.546 en Adam, 1.800 en Biz/ed, 1.949 en GeoGuide y 4.579 en $\mathrm{Omni}^{21}$; Bubl e Infomine, que abarcan todas las áreas temáticas, incluían unos $12.000 \mathrm{y}$ 20.000 respectivamente.

\section{«Actualmente las bibliotecas ya integran en el catálogo la des- cripción de las bases de datos y de las revistas digitales comer- ciales, pero continúan ordenan- do páginas web por el título o temáticamente»}

Algunos servicios especifican el tipo de documentos que recopilan. Por ejemplo, Sosig incluye artículos, informes, bases de datos bibliográficas, bibliografías, libros, organizaciones, datos, materiales educativos, FAQs, publicaciones oficiales, organismos oficiales, revistas, listas de distribución, news, obras de consulta (diccionarios, etc.), proyectos de investigación, guías de recursos y programas informáticos. Infomine indexa todo tipo de publicaciones seriadas electrónicas, news, bases de datos bibliográficas y de imágenes, conjuntos de datos, directorios, bibliotecas virtuales, índices, sitios web, guías temáticas, obras de referencia, libros de texto, manuales y materiales educativos de todo tipo, buscadores, mapas, etc.

En el caso de los recursos web que se integran en el catálogo, uno de los valores más sólidos de InterCAT, NetFirst y Corc es que los documentos que incluyen han sido seleccionados por profesionales atendiendo a los objetivos de las propias bibliotecas y a las necesidades de sus usuarios. Los criterios de selección utilizados son paralelos y muy similares a los usados en las bases de datos anteriores. En general, se centran en el contenido, alcance, diseño, facilidad de uso, revisiones y reseñas disponibles así como en los objeti- 
vos y destinatarios del recurso; cuando se trata de fuentes comerciales también se considera su coste. Con relación al tipo de materiales informativos que se incluyen en el catálogo, Weber (1999) apunta que la mayoría de bibliotecas opta por descartar los más efímeros, la literatura gris de empresas y asociaciones, las páginas que no tienen un apoyo institucional, etc. En general, como dice la autora, hay una cierta tendencia a catalogar sólo aquellas páginas que son responsabilidad de la biblioteca o de la institución de la que depende o las que tienen carácter institucional siempre que exista el compromiso de actualizarlas ${ }^{21}$.

Una de las diferencias con las bases de datos de recursos web es que, cuando la descripción de estos materiales se integra en el catálogo, su selección se ha de inscribir en la política general de desarrollo de la colección del centro. Se entiende que esta política está recogida en "un documento escrito que define el alcance y la naturaleza de las colecciones que tiene la biblioteca y las políticas y planes para continuar el desarrollo de las mismas, con indicaciones precisas de los puntos fuertes actuales, su intensidad actual en áreas temáticas relevantes, así como la mención de la filosofía de selección en relación con las metas de la institución, los criterios generales de selección y la libertad intelectual" (Guide to review, 1991, p. 28). La Association for Library Collections and Technical Services ha publicado una serie de guías con directrices sobre los distintos aspectos de la gestión de colecciones; la Guide for written collection policy statements (1996) es la que establece cuál ha de ser el contenido del documento de políticas de desarrollo de la colección. En el apartado de recursos digitales hay que tener en cuenta los Collection development policy statement for electronic formats (1994) que incluyen criterios para la su selección. En las bases de datos de páginas web su política de desarrollo no acostumbra a estar tan formalizada; sin embargo se puede considerar que viene determinada por sus objetivos, temática y los usuarios a los que se dirige.

\section{Descripción}

Aunque en algunos casos las descripciones que se utilizan en estas bases de datos parten de los modelos bibliotecarios utilizados en la catalogación tradicional (como Isbd o Aacr2), en general no son tan completas ni uniformes como las de los registros en formato Marc de los catálogos de biblioteca. De hecho, hasta el momento no se han regido por unos criterios comunes básicos que den directrices sobre la descripción y que identifiquen los elementos mínimos que han de configurar el registro. Estos servicios de recursos web son muy similares a las bases de datos científico-técnicas tradicionales que en el este apartado no son precisamente un ejemplo de normalización y homogeneidad.

Dos son los niveles de descripción que predominan. El más elemental sólo incluye el título, la dirección electrónica y a menudo un resumen (Agnic, Argos, Gateway to online resources y MEL son ejemplos de este tipo). En un segundo nivel, se encuentran otras descripciones más completas que incluyen autor, título, URL, código de clasificación y resumen; en algunos casos también se anotan palabras clave, materias de alguna lista o tesauro, país, lengua, etc. La mayoría de los servicios anotados en la tabla 1 se incluirían en esta categoría. Dutchess, Sosig, The scout report signpost, Bubl, The internet public library e Infomine son, entre otras, ejemplos de bases de datos que dan descripciones bastante completas de las fuentes informativas indexadas.

Muchas de las bases de datos del Reino Unido siguen de forma más o menos estricta las Roads cataloging guidelines (1999) ${ }^{23}$, que incluyen un conjunto muy completo de elementos y que además han desarrollado diversos formularios adecuados a los distintos tipos de recursos y servicios susceptibles de catalogación. Entre los formularios impulsados se presta atención al $D C$, conjunto de metadatos que se está implantando como estándar para describir sitios web en internet. Las bibliotecas nacionales de los países nórdicos (a través del Nordic metadata project) y la National Library of Australia, entre otras, también estimulan las propuestas destinadas a impulsar el uso del sistema de metadatos $D C$.

\section{«Los registros de Corc tienen el doble objetivo de alimentar ca- tálogos y guías o bases de da- tos de fuentes de internet»}

En marzo de 1995 Oclc y el National Center for Supercomputing Applications organizaron el Oclc/Ncsa metadata workshop con la participación de representantes de la Internet Engineering Task Force y de expertos en biblioteconomía, informática, codificación de textos y áreas relacionadas. El objetivo del seminario era definir un conjunto de elementos de metadatos para describir recursos internet que recibió el nombre de Dublin core. Entre julio y agosto de 2000 hubo un período de votación para aprobar este conjunto de metadatos como norma Ansi Z39.85 (Draft, 2000).

La descripción de fuentes web para su integración en el catálogo tiene la ventaja de poder prescindir de soluciones locales, ya que se aplican los mismos estándares que al resto de materiales. Esta opción asegura el uso de una normativa que se irá adaptando a me- 
dida que sea necesario. Disponemos ya de una Isbd(Er) actualizada que contempla todo tipo de recursos $w_{e b}{ }^{24}$, y sendos comités trabajan intensamente en la puesta al día de la Isbd(S) (Parent, 1999) y del capítulo 12 de las Aacr2r para acomodar los dos textos a la catalogación de publicaciones seriadas electrónicas de acceso remoto (Hirons, 2000). Paralelamente también se está llevando a cabo una revisión global del código Aacr ${ }^{25}$.

Los estándares de descripción utilizados en los proyectos InterCAT y Corc son las Aacr 2 y el formato Marc. Además, las bibliotecas que han participado en los dos proyectos han tenido al alcance los instrumentos necesarios (listas de autoridades de nombres) para realizar el control de los puntos de acceso bibliográficos. Obviamente, poder usar esas herramientas agiliza el procesamiento de los recursos y asegura que los puntos de acceso a la descripción sean coherentes con los del resto de registros. Corc también permite la exportación e importación de registros en formatos distintos a los tradicionales en catálogos. En concreto, el sistema de metadatos $D C$ está consiguiendo la categoría de estándar en este campo y Oclc es una de las instituciones que lideran esta iniciativa.

Así pues, el $D C$, conjunto de elementos de metadatos mucho más reducido que el resultante de aplicar Aacr2 y Marc (se limita sólo a 15) representa el punto en el que pueden converger los dos modelos: creación de bases de recursos web e integración de éstos en el catálogo. Por un lado, el $D C$ prevé la posibilidad de utilizar en determinados campos (autor, materias, contribuidor, etc.) información procedente de listas de autoridades de nombres y materias, clasificaciones y tesauros bibliotecarios; por otro, a partir de las tablas de correspondencias entre los formatos Marc y $D C$, se pueden convertir fácilmente los registros de uno y otro. Corc está demostrando que un mismo registro, ya esté en Marc o en $D C$, puede utilizarse para alimentar bases de datos y catálogos. Cuando el $D C$ sea aprobado como una norma las bases de datos tendrán finalmente un estándar de descripción que asegurará una compatibilidad mínima de los registros y facilitará la interoperabilidad entre sistemas.

\section{Indización y clasificación}

Algunas de las bases de datos mencionadas utilizan las listas bibliotecarias de encabezamientos de materia para indizar los documentos que incluyen. Infomine y Scout report signpost usan la lista Lcsh; Omni, el Medical subject headings (MeSH). Su uso facilita la consulta de los índices de materia y permite reproducir en las bases de datos de webs la estructura sindética (interrelacionada) que caracteriza el catálogo de bi- blioteca y que tan útil es para encontrar documentos relacionados con el área objeto de la consulta. Las tres guías anteriores tienen esta capacidad.

En otros casos la indización se hace a partir de diversos tesauros. Sosig utiliza una adaptación de Hasset (Humanities and social science electronic thesaurus) y Adam, el Art \& architecture thesaurus. Estas dos bases de datos permiten la consulta de los índices de descriptores, pero no enlaza los recursos relacionados unos con otros.

Un tercer caso sería el de las bases de datos que no incluyen la materia de los recursos (Argos, Gateway to online resources y $M E L$ ). Finalmente, algunos servicios utilizan tanto listas de materias como tesauros y/o palabras clave: la Internet public library estaría en este apartado. La página web Beyond bookmarks (McKiernan, 2000) es un directorio de guías de webs que han adoptado esquemas formales de clasificación y/o vocabularios controlados. En ella se puede observar una serie de guías que utilizan estos vocabularios en la indización de las fuentes que incluyen.

\section{«Hay una cierta tendencia a ca- talogar sólo aquellas páginas que son responsabilidad de la biblioteca o de la institución de la que depende, o las que tienen carácter institucional siempre que exista el compromiso de ac- tualizarlas»}

El uso de clasificaciones bibliotecarias en esas bases de datos se encuentra también muy generalizado. Una gran mayoría de las experiencias analizadas dispone de una clasificación que se utiliza como punto de acceso en la consulta y en la organización de los recursos. Los beneficios del uso de un esquema de clasificación en las búsquedas está fuera de toda duda; diversos autores señalan sus ventajas: simplifica la consulta a los usuarios poco experimentados; permite ampliarlas o limitarlas; sitúa las materias dentro de un contexto; facilita el acceso multilingüe, ya que la notación es independiente del idioma, etc. (Lim, 1999; Vizine-Goetz, 1996).

Entre las clasificaciones generales universales destacan: DDC (Adam, Bizled, Bubl, Internet public library), CDU (Sosig), Library of Congress classification (LCC) (CyberStacks, The scout report signpost). Algunas bases de datos usan clasificaciones generales nacionales (Dutchess), clasificaciones especializadas en un área temática (Omni, que como se ha dicho utiliza la clasificación MeSH de la National Library of Medicine), o han desarrollado esquemas propios (Eevl, 
Eels, Librarians index to internet). Beyond bookmarks, ya citada, incluye una amplia lista de guías y bases de datos de webs que utilizan clasificaciones.

Las bibliotecas que han participado en los proyectos InterCAT y Corc de Oclc han utilizado los sistemas de materias habituales en cada institución (Oclc no daba instrucciones al respecto). Es obvio que en el apartado de vocabularios controlados, la Lcsh es el sistema más utilizado en estos proyectos mientras $L C C$ y $D D C$ lo son en el apartado de las clasificaciones. En NetFirst se asignan materias de la $L c s h$ y se usa la $D D C$ como esquema de clasificación. Además, Corc ha puesto a disposición de las bibliotecas participantes las versiones electrónicas de la $D D C$, de la $L c s h$ y de otros tesauros como $\mathrm{MeSH}$ y también varios instrumentos que facilitan la asignación automática de palabras clave y de números de la $D D C^{26}$. Clasificar es un proceso lento y costoso y es por ello que distintos proyectos (Corc y también los que van más destinados al desarrollo de portales temáticos) investiguen procedimientos y desarrollen tecnología que permita la clasificación automática de los sitios web.

\section{Recuperación de la información}

Todas las experiencias analizadas permiten acceder a los registros a partir de cualquiera de los campos determinados a priori. Para poder gestionar las referencias de los webs es necesario disponer de algún sistema de gestión de bases de datos (sgbd) que se ocupe de su creación y explotación ${ }^{27}$. Por otro lado, hay que disponer de otras aplicaciones que faciliten la consulta de los registros desde el web y la interoperabilidad con otros sistemas, entre otras necesidades. En este sentido, una de las plataformas más utilizadas por las bases de datos de recursos web es $\operatorname{Roads}^{28}$, un paquete gratuito y personalizable que dispone de diversos módulos: una CGI para poder comunicar el sgbd con el entorno web, comprobador automático de enlaces, elaboración de estadísticas, interfaz para la catalogación y validación de registros, desarrollados en lenguaje Perl y que permiten gestionar bases de datos de descripciones de recursos y facilitar su consulta desde el web.

Entre los sistemas llevados a cabo en el ámbito bibliotecario hay que destacar Mantis $^{29}$, utilizado en el proyecto Corc. Es una aplicación para sistemas de catalogación web desarrollada a partir de diversos proyectos de investigación y que utiliza $R D F \mathrm{y} \mathrm{xml}$ para dar apoyo a la gestión de metadatos. Tiene capacidades parecidas a los programas que gestionan, compilan e indexan bases de datos de webs y facilita además un control de las autoridades (nombres, materias y clasificaciones) con los instrumentos bibliotecarios tradicionales.
Algunos servicios posibilitan la recuperación no tan sólo de la referencia sino del texto completo de los documentos. Esta opción es más onerosa que la anterior ya que se requiere un motor de búsqueda (o sistema de indexación) que es más costoso y unos equipos informáticos de mayor capacidad y potencia. Uno de estos motores, Combine harvester ${ }^{30}$, fue desarrollado en el marco del proyecto Desire y se comercializó en 1998 —entre otros servicios lo utilizan Eels y NWI (Nordic web index) - É Éste es un sistema de arquitectura abierta que funciona en entornos Unix, está escrito en Perl y se basa en el modelo cliente-servidor; esencialmente, está formado por los siguientes componentes: Cabin (controlador centralizado del sistema); Harvester (robot que se ocupa de la indexación automática); $H D B$ (sgbd de la base de datos de webs). También se puede citar Index $+{ }^{31}$, utilizado actualmente por Adam para facilitar la consulta del texto completo de los recursos, por la Scottish cultural resources access network ${ }^{32}$ y por diversas galerías y museos; este sistema permite gestionar todo tipo de información, tiene estructura cliente-servidor y dispone tanto de pasarelas web como de Z39.50. Otro motor de búsqueda, el de Excite, es utilizado por MEL.

\section{"La descripción de recursos web para su integración en el catálogo tiene la ventaja de po- der prescindir de soluciones lo- cales, ya que la normativa con- templa su descripción y se les pueden aplicar los mismos es- tándares que al resto de mate- riales»}

Hay que destacar que muchos de los procedimientos citados permiten consultar las fuentes seleccionadas a partir de una clasificación, cuyos códigos (que se pueden extraer automáticamente de los registros) son utilizados para organizar la estructura jerárquica del servicio. Esta opción, inexistente en muchos de los programas que gestionan los catálogos de biblioteca, pasa a tener un lugar muy destacado en las bases de datos de webs.

\section{Mantenimiento}

Aunque no existe un modelo único para la planificación y el mantenimiento de las pasarelas temáticas, una fórmula que utilizan algunos de los servicios descritos es la de configurar dos equipos de trabajo con tareas y funciones distintas. Por un lado es frecuente la existencia de un consejo editorial que se ocupa de marcar las líneas estratégicas de la base de datos, de decidir el desarrollo y la creación de nuevos servicios, de 
obtener financiación, etc. Por otro hay comités técnicos que se encargan de las cuestiones relacionadas con su mantenimiento, tales como la selección y el análisis de los sitios, la recuperación y la difusión. En la mayoría de los casos existe algún canal, correo electrónico, formulario web, etc., para facilitar que cualquier usuario pueda proponer la inclusión de nuevos recursos. Las propuestas, que se tratan de igual forma que las desideratas de la biblioteca tradicional, son analizadas por el comité técnico encargado de decidir en último término la conveniencia de su inclusión final.

La tarea de mantenimiento de los recursos web integrados en el catálogo es paralela a la que se acaba de describir para los portales temáticos. Tradicionalmente las bibliotecas han tenido personal encargado de las políticas de colección y de la selección de materiales. En general son los bibliotecarios de desarrollo de la colección, o la figura del bibliógrafo, los que se encargan de la selección de webs. Ahora bien, en algunos centros se han designado responsables de la colección digital. En todo caso, esta asignación de responsabilidades dependerá de cada organigrama concreto.

Uno de los principales problemas del mantenimiento está relacionado con la necesidad de actualizar continuamente la información (control de cambios de dirección o desaparición del sitio). Relacionados con este aspecto, existen programas informáticos que permiten comprobar la vigencia de las URL y resuelven, con relativa sencillez, esta incomodidad. En el proyecto Corc, su mantenimiento se lleva a cabo por medio del servicio Purl, una dirección intermedia que asegura la localización del recurso.

\section{Tendencias}

El cuadro dibujado hasta aquí explica el detalle de la organización y el funcionamiento de los dos modelos básicos que facilitan el acceso a los recursos web desde las bibliotecas. Para completar la panorámica se enumeran y comentan a continuación las tendencias actuales en este ámbito.

\section{a. Cooperación}

Una buena parte de las bases de datos citadas se han elaborado en el marco de organizaciones que facilitan fórmulas de cooperación y de colaboración con la finalidad de optimizar los medios disponibles y mejorar los servicios. La reciente constitución de IMesh, Renardus y RDN es un buen ejemplo de ello. A partir de estos proyectos cooperativos a veces se agrupan centros de distintos países especializados en un área temática determinada (Agnic, Novagate y Eels por ejemplo); en otras ocasiones se trata de proyectos de ámbito nacional entre gateways que se ocupan de áre- as temáticas distintas (éste sería el caso de $R D N$ en el Reino Unido o de $S s g-F i$ en Alemania).

En lo que respecta a la formación de catálogos, la cooperación es también uno de sus rasgos distintivos. A través de servicios nacionales, de catálogos colectivos y de servicios comerciales, las bibliotecas han utilizado los registros bibliográficos procedentes de fuentes muy diversas y han compartido e intercambiado información bibliográfica. Los registros de recursos electrónicos no constituyen una excepción a esta tendencia, y los mismos proyectos de Oclc no se habrían podido llevar a cabo sin la colaboración de numerosas bibliotecas. La actual formación de catálogos no se entiende sin este movimiento de información bibliográfica.

\section{b. Interoperabilidad}

Una buena parte de las investigaciones que se realizan en el marco de los portales temáticos tiene el objetivo de facilitar su interoperabilidad, esto es, la consulta simultánea de diversas bases de datos con la obtención de un listado único y acumulativo de resultados. En los proyectos Roads y Desire se trabaja en este sentido y $R D N$ incorpora un motor de búsqueda que facilita la consulta sincrónica en sus diversos catálogos temáticos ${ }^{30}$. También se pueden efectuar consultas simultáneas a Eels (Suecia) y a Eevl (Reino Unido), dos bases de datos especializadas en ingeniería y elaboradas con criterios de calidad similares aunque residen en distintos países ${ }^{34}$.

Los problemas que hay que resolver para que las investigaciones sigan avanzando y la interoperabilidad sea un hecho son complejos y tienen que ver especialmente con los contenidos de los registros pero también con la tecnología. Los servicios que se han ido creando disponen de modelos de descripción muy distintos (muchos parten del $D C$, pero todavía hay muy poca uniformidad) y usan una gran diversidad de instrumentos para su clasificación e indización. La interoperabilidad sólo puede ser efectiva si existe un alto grado de coordinación y colaboración entre los diversos servicios.

En lo que respecta a los aspectos tecnológicos hay que recordar que la mayoría de las experiencias descritas han desarrollado sistemas de recuperación de la información que no pueden trabajar coordinadamente entre sí. Se necesita una arquitectura global que especifique cuáles son los componentes individuales y cómo se han de comunicar. Los programas con una estructura bien definida son más fáciles de mantener, ampliar y reutilizar. El principal objetivo del proyecto IMesh toolkit no es otro que precisar, implementar y probar esta arquitectura. Iniciado en septiembre de 1999, tiene por objetivo poner las bases para el desa- 
rrollo de software para pasarelas temáticas y hacerlo de manera que se reduzcan los costes iniciales de la puesta en funcionamiento de un servicio de estas características. Se puede consultar el documento IMesh toolkit IDL project proposal que especifica con más detalle estas cuestiones en la dirección web indicada. La interoperabilidad también es uno de los principales objetivos del proyecto Renardus descrito en apartados anteriores.

\section{http://www.imesh.org/toolkit/proposal}

En el entorno bibliotecario la existencia de catálogos colectivos es una fórmula centenaria para dar acceso a colecciones diversas y distantes. Ya en los años noventa se han desarrollado otros mecanismos, totalmente operacionales, que facilitan la consulta de bases de datos separadas a partir de una única interfaz y la obtención de resultados unificados. El protocolo Z39.50 $0^{35}$ (que facilita esta consulta simultánea) empieza a tener un uso bastante generalizado y son diversos los proyectos de investigación que muestran su potencial $^{36}$. Corc prepara la puesta en marcha del acceso Z39.50 a la base de datos para facilitar la recuperación y la exportación de registros desde clientes Z39.50. En este proyecto el uso de los estándares $R D F$ y xml también facilitará la interoperabilidad de diversas bases de datos.

En lo que respecta al contenido de los registros, que puede dificultar el acceso y la consulta simultánea a diferentes catálogos, hay que subrayar que los registros bibliotecarios siempre han sido bastante uniformes y normalizados en cuanto a los datos bibliográficos que se anotan. En el apartado de las materias el grado de normalización no es tan grande y constituye una notable dificultad tanto en la opción de integrar la descripción de recursos web en el catálogo como en la de elaborar guías.

\section{c. Uso de registros con funciones diversas}

Es obvio que los portales temáticos tienen un papel por su inmediatez en el momento de buscar directamente fuentes electrónicas; pero también lo es que los recursos seleccionados, o al menos una parte, han de estar integrados con toda la colección de materiales que la biblioteca pone a disposición de su comunidad de usuarios y que el lugar natural de esta integración ha de ser el catálogo. Por otro lado, elaborar y mantener tanto registros de catálogo como guías o bases de datos de fuentes web es una carga adicional para el personal bibliotecario cada vez más escaso y/o con más atribuciones. Es por ello que, entre las tendencias detectadas en el ámbito de las bibliotecas, destaca además de la cooperación, la interoperabilidad de sistemas y los desarrollos tecnológicos que también ca- racterizan las pasarelas temáticas- el uso de registros con funciones diversas (para alimentar catálogos y para elaborar guías de recursos).

Para evitar la doble carga de tener que mantener dichas guías y registros en el catálogo recientemente muchas bibliotecas individuales han desarrollado nuevos modelos de guías web, sobre todo para revistas electrónicas que usan el registro Marc como localización única para almacenar y mantener las URLs de las revistas electrónicas accesibles desde el catálogo y desde páginas web. De hecho el proyecto Corc sigue el hilo de estas iniciativas y las amplía a toda clase de recursos web. Uno de sus objetivos es investigar cómo los mismos metadatos pueden servir para alimentar el catálogo y para elaborar guías. Además, para que todo esto sea posible, hay que usar instrumentos comunes y normalizados; el catálogo dispone de ellos desde hace muchos años. En lo que respecta a los metadatos que se utilizan para elaborar bases de datos de webs, el $D C$, que se está imponiendo como el estándar más aceptado, permite el uso de listas y tesauros normalizados y clasificaciones bibliotecarias para su indización.

\section{A modo de conclusión}

Para finalizar, se presentan unas consideraciones que puedan servir de orientación para las bibliotecas que quieren abordar la organización de los recursos web y no tienen claro el camino a seguir ante la disyuntiva que plantea la decisión sobre la inclusión o no de las descripciones de los recursos web en el catálogo.

Las ventajas de esta integración ya han sido destacadas anteriormente: una política de desarrollo de la colección y unos principios de selección uniformes para todos los materiales sean del tipo que sean; unos registros normalizados, tanto en lo que se refiere al formato como al contenido, que facilitan la cooperación y el intercambio; un único instrumento de recuperación, el catálogo, que asegura que cada documento individual se relacione con el resto de la colección. A la larga, ésta tendría que ser la solución definitiva para todos los recursos web gratuitos que responden a la política de desarrollo de la colección y a los criterios de selección de los centros y que tienen garantías de permanencia.

En las actuales circunstancias, y atendiendo al carácter experimental de la organización de estos documentos, optar por una base de datos separada que tan sólo contenga registros de webs gratuitos tiene también unas ventajas: los sistemas de recuperación de la información que se han desarrollado acostumbran a tener más prestaciones que los catálogos en línea usados por la mayoría de las bibliotecas; la creación de una herramienta separada o segregada del catálogo, que 
tendría muchas similitudes con los portales y localizadores de internet muy conocidos y utilizados por los usuarios de la Red, facilitaría su promoción; y, finalmente, el usuario de una base de datos dedicada exclusivamente a recursos web sabe que tiene a su alcance, de manera inmediata, todos los materiales que encuentra descritos sin necesidad de tener que desplazarse a la biblioteca para poderlos consultar.

Ahora bien, el catálogo ha tenido tradicionalmente unas características que lo han convertido en un instrumento óptimo para la recuperación de la información y que han facilitado el intercambio de registros. Por ello, la elaboración de una base de datos separada del catálogo debería partir, como condición previa, de la normalización de las descripciones y del uso de los instrumentos estándar de indización y clasificación. Seguir estas directrices aseguraría la transferencia de registros desde esta base de datos a los catálogos locales de las bibliotecas y, en el caso de disponer de un cliente-servidor Z39.50, la consulta simultánea y uniformizada del catálogo y de la base de datos de recursos web.

El análisis realizado en este estudio pone en evidencia el creciente interés manifestado principalmente por parte de muchas bibliotecas nacionales y universitarias europeas, americanas y australianas, para organizar los recursos web y ponerlos al alcance de sus usuarios. Se han descrito y analizado muchas experiencias entre las cuales, lamentablemente, no se encuentran ejemplos españoles. Tampoco se encuentra presencia española alguna entre los participantes de las distintas iniciativas europeas que se han creado para promover este ámbito (Desire, Renardus e IMesH). Si las bibliotecas españolas quieren mantenerse en línea con los proyectos de los países más punteros han de destinar esfuerzos y medios para promover acciones, sobre todo cooperativas, que faciliten a sus usuarios el acceso a las fuentes web.

\section{Notas}

1. Resumen y adaptación del informe encargado por el Consorci de Biblioteques Universitàries de Catalunya (Cbuc) y realizado mediante un convenio con la Fundació Bosch Gimpera, entregado en enero de 2000. Las direcciones electrónicas de los documentos y servicios citados se han revisado en julio de 2000.

2. El proyecto Decomate del Servei de biblioteques de la Universitat Autònoma de Barcelona es un ejemplo de una colección de publicaciones digitales instaladas en un servidor local. Incluye 372 revistas científicas suministradas en formato electrónico por diversos editores (Elsevier Science, Kluwer Academic Publishers y la propia Universitat Autònoma de Barcelona). http://decomate.uab.es

3. En algunos casos estas bases de datos son generales; esto es, incluyen documentos de todas las áreas temáticas y pueden ir dirigidas al público de bibliotecas universitarias o públicas. Entre los ejemplos de portales de carácter general destacan Dutchess y Bubl.

4. Para completar la descripción y análisis de los subject gateways puede consultarse el Desire information gateway handbook (Desire, 1990), un manual que analiza con detenimiento los aspectos estratégicos, de contenido y también las cuestiones tecnológicas de este tipo de bases de datos. Otro texto, no tan extenso, pero con un propósito parecido es el artículo "Quality-controlled subject gateways: definition, typologies, empirical overview" (Koch, 2000).

5. Actualmente el proyecto IMesh elabora un censo que incluirá la descripción de estas bases de datos: IMesh: international collaboration on internet subject gateways.

http://www.imesh.org

6. El programa eLib (Electronic libraries programme) tiene sus orígenes en un informe de Brian Follett presentado en 1993 al Jfclr group en el que se ponía de manifiesto la importancia de las tecnologías de la información para el desarrollo futuro de los servicios bibliotecarios. Como resultado en 1995 se creó el programa $e$ Lib (gestionado por el Jisc, Joint information systems committee).

http://www.ukoln.ac.uk/services/elib

7. La Resource discovery network empezó a funcionar en enero de 1999 (su presentación oficial se hizo en septiembre del mismo año). Tiene financiación para tres años del Jisc y de los Higher education funding councils entre otros organismos.

http://www.rdn.ac.uk

8. Desire. En la segunda fase del proyecto participan instituciones del Reino Unido, los Países Bajos, Suecia, Dinamarca y Noruega. Para más detalles véase, además del sitio web, Worsfold (1999).

http://www.desire.org

9. Véase la nota 5.

10. Información más detallada sobre el proyecto Renardus: http://www.renardus.org

11. Australian subject gateways to internet resources. http://www.nla.gov.au/pathways/pthw_global.html

12. Los usuarios tienen la seguridad que los sitios web que encuentran a partir del catálogo tienen el sello de garantía de la biblioteca. Hay indicios de que tanto los catalogadores como los bibliotecarios de referencia y de desarrollo de la colección muestran su preferencia por el concepto one-stop shopping y por que el catálogo sea el instrumento de acceso a todos los materiales seleccionados (Porter, 1999).

13. La Biblioteca Digital del Consorci de Biblioteques Universitàries de Catalunya y las colecciones de revistas digitales de la mayoría de las bibliotecas de las universidades catalanas siguen esta doble opción. Otros centros españoles sólo ofrecen de momento listas de títulos u ordenadas por materias de sus colecciones digitales a través de una página web que, a veces, tiene su acceso restringido a las direcciones IP del campus en cuestión. http://www.cbuc.es

14. En la página web siguiente se puede comprobar el funcionamiento de este modelo:

-Los Alamos National Laboratory. Electronic journals. Lanl, c1998, 15 enero de 1999. Consultado en: 10-07-00.

http://lib-www.lanl.gov/cgi-bin/ejrnlsrch.cgi

-Un modelo parecido es el de la biblioteca de la University of California, San Diego:

University of California, San Diego. Libraries. Electronic journals, newsletters, etc. General browsing. [La Jolla, California]: Ucsd, última actualización: 20 de octubre de 1999. Consultado en: 10-07-00. http://gort.ucsd.edu/ejourn/index.html 
Si sus documentos son estratégicos para su compañia, también lo son para nosotros

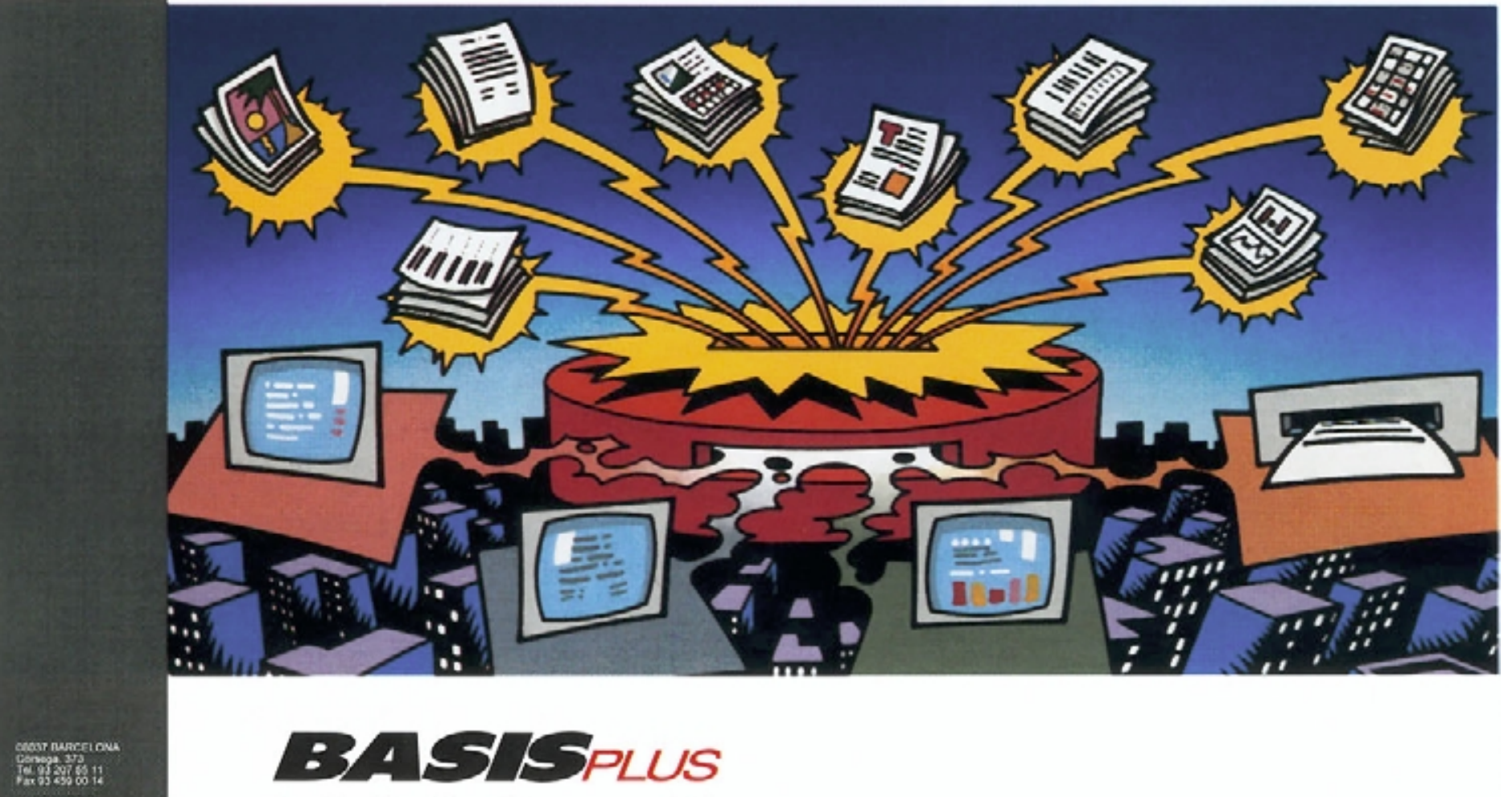

2005mpar

2000 grese 25.104

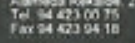

Jigospenpon

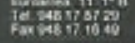

tropvarmen

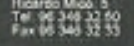

cec

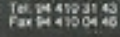

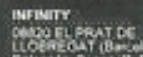

toming

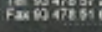

soc

Tixighas

centesuscomivar

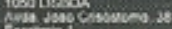

18.

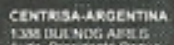

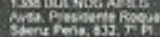

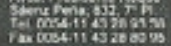

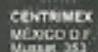

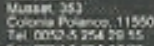

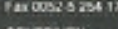

GeNTRNer

ching atoms

and

centresa.colounat

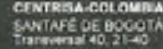

centrisa-anase

$\cos _{R+R^{2}}^{R \cup D}$

\section{La Solución Documental}

BASISplus es el único sistema de gestión de documentos que permite gestionar de un modo integrado toda la información que circula por su organización.

Con BASISplus su trabajo será más fácil, su tiempo más rentable y su información más segura y accesible.

\section{TECHLIBPLUS} Más de 1 millón de usuarios,
2.500 instalaciones en 75 paises y en 24 idiomas

\section{La Gestión de Bibliotecas}

TECHLIBplus es una solución completa totalmente diseñada bajo entorno Web para gestionar los recursos de información y actualizar el trabajo diario de las bibliotecas.

Aplicaciones basadas en las tecnologias BASISplus y TECHLIBplus:

Centros de Documentación

Sistemas de Gestión de Calidad

Gabinetes de Prensa

Difusión de Boletines Oficiales

Gestión de Expedientes

Normativa y Procedimientos

Producción Editorial

Gestión de Archivos

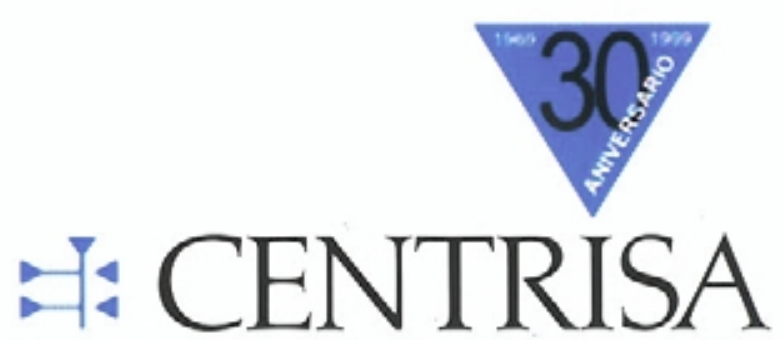

Tecnologias de la Información 
15. InterCat ha sido una base de datos operativa hasta finales de 1999.

16. La siguiente página web da información sobre este proyecto histórico y acceso a los trabajos presentados al coloquio que se organizó sobre la materia y a Cataloging internet resources: a manual and practical guide, ed. by Nancy B. Olson, que se elaboró para orientar a los bibliotecarios participantes. Consultado en: 20-07-00.

http://www.Oclc.org/Oclc/research/projects/intercat.htm

17. La correspondencia entre los campos y subcampos del formato de NetFirst y los del formato Usmarc, así como datos de la estructura y capacidades de recuperación de la base de datos NetFirst están bien especificados en: http://www.Oclc.org/Oclc/man/z39.50/netfirst.htm

18. Página de acceso a la documentación sobre Corc: http://www.oclc.org/oclc/corc/index.htm

19. Las bibliotecas de las universidades de La Rioja y Politécnica de Valencia y la del Cindoc han participado en Corc.

20. Corc adopta el término pathfinder para denominar a los portales temáticos, coincidente con el que tradicionalmente ha designado a las guías impresas de recursos bibliotecarios sobre un tema en las bibliotecas norteamericanas.

21. Entre enero y julio de 2000 cada una de estas bases de datos apenas aumentó en unas pocas decenas de registros.

22. El servicio AcqWeb ofrece acceso a documentos sobre desarrollo de la colección y selección de materiales en general y aplicados a sitios web. Para ejemplos concretos de criterios de selección de bibliotecas que incluyen la descripción de recursos web en el catálogo, véanse las directrices de la University of Rochester y de la Yale University en la bibliografía.

23. El proyecto Roads, subvencionado dentro del programa eLib, tiene como objetivos el desarrollo de software para la gestión de portales temáticos, la investigación para facilitar la consulta simultánea y la interoperabilidad entre distintas bases de datos y el desarrollo de estándares de indización, catalogación y búsqueda de recursos web.

24. Con relación a la descripción de fuentes electrónicas remotas, actualmente la Isbd(Er) está mucho más actualizada que el capítulo 9 de las Aacr2. Sin embargo, el Joint steering committee for revision of Aacr ha tomado ya la decisión de actualizar este capítulo y armonizarlo con la Isbd(Er): "Outcomes of meeting held in Brisbane, Australia 18-20 octubre 1999”. En: Announcements. Joint steering committee for revision of Anglo-American cataloguing rules, 20 de noviembre de 1999. Consultado en: 24-07-2000.

http://www.nlc-bnc.ca/jsc

25. En la página web del Joint steering committee for revision of AngloAmerican cataloguing rules hay información de las decisiones que se toman con relación a la revisión del código.

http://www.nlc-bnc.caljscl

26. NetFirst y Corc han dado prioridad a la $D D C$ porque Oclc forest press es propietaria de esta clasificación, pero también porque su estructura jerárquica es óptima para la organización y recuperación de materiales; de hecho, la $D D C$ es el esquema de clasificación más utilizado para la organización de guías de recursos (McKiernan, 2000).

27. Uno de los programas utilizados es Oracle (por ejemplo, Dutchess), que ya se ha convertido en un referente de los sistemas de gestión de bases de datos.

28. Adam (hasta 1997), Biz/ed, Eels, Novagate, Sosig y Omni son algunas de las bases de datos que utilizan o han utilizado Roads.

29. Mantis [Dublin, Ohio: Oclc], última actualización: 29 de marzo de 2000. Consultado en: 27-07-00.

http://orc.rsch.oclc.org:6464/
30. Lundberg, S. The combine harvesting robot. NetLab, última modificación: 30 de febrero de 2000. Consultado en: 27-07-00.

http://www.lub.lu.se/combine

31. Se puede obtener más información en: http://www.ssl.co.uk/indexx/indexx/html

32. Consultable en:

http://www.scran.ac.uk

33. Véase el buscador Resource finder en la página principal de $R D N$. http://www.rdn.ac.uk

34. En la dirección siguiente se puede probar el sistema: http://roads.ukoln.ac.uk/eels-eevl

35. Z39.50 es un estándar Ansi que también tiene un paralelo en la norma ISO 23950 .

36. El proyecto Bopac2, desarrollado por el Department of Computing de la University of Bradford con una ayuda del British Library Research and Innovative Centre, permite la consulta simultánea de numerosos catálogos de grandes bibliotecas — Library of Congress, British Library y el catálogo colectivo Melvyl de las bibliotecas de la University of California, entre otros- Este catálogo experimental se puede consultar en la siguiente dirección:

http://www.comp.brad.ac.uk/research/database/bopac2.html

\section{Bibliografía}

Abadal, Ernest; Estivill, Assumpció. "L'accés als recursos web des de les biblioteques: informe encarregat pel Consorci de Biblioteques Universitàries de Catalunya". Barcelona, enero 2000, 55 pp. http://www.cbuc.es/5digital/55docs.htm

AcqWeb's library \& information science resources: collection development sites. AcqWeb, c1994-2000. Consultado en: 25-07-00.

http://www.library.vanderbilt.edu/law/acqs/lis_cd.html

Aguillo, Isidro. "Indicadores hacia una evaluación no objetiva (cuantitativa) de sedes web". En: VII jornadas españolas de documentación. La gestión del conocimiento: retos y soluciones de los profesionales de la información. Bilbao: Universidad del País Vasco, 2000, pp. 233-248.

Argudo, Sílvia; Núñez, Lluïsa. "Les biblioteques universitàries catalanes a la web”. En: Item, 1997, enero-junio, n. 20, pp. 6-22.

Beumala, Àngel et al. "Base de datos de recursos internet científico-técnicos: Ep! (enlaces politécnicos).” En: VI jornadas españolas de documentación: los sistemas de información al servicio de la sociedad. Barcelona: Cobdc, 1998, pp. 149-156.

http://www.upc.es/catala/biblio/biblio.htm

Brickley, Dan; Kirriemuir, John; Tredgold, Jasper. "Prototype integrated Sbig/Index interface". En: Desire-Development of a European service for information on research and education. Consultado en: 27-07-00. http://www.sosig.ac.uk/desire/index/integration.html

Codina, Lluís. "Búsqueda y obtención de recursos digitales de interés". En: Revista española de documentación científica, 1998, v. 21, n. 3, pp. 328-334.

Codina, Lluís. "Evaluación de recursos digitales en línea: conceptos, indicadores y métodos". En: Revista española de documentación científica, v. 23 , n. 1,2000 , p. $9-44$.

Codina, Lluís. "Evaluación, descripción y representación de recursos digitales". En: Documentación digital: curso de postgrado impartido a distancia a través de internet, 1999, julio, v. 1. Barcelona: Universitat Pompeu Fabra, 1999. 
Collection development policy statements for electronic formats. Rev. febr. 1994. Reference and user services association, Collection development \& evaluation section, Collection development policies committee, febrero, 1994.

Corc: cooperative online resource catalog. Oclc. Consultado en: 26-07-00. http://www.oclc.org/oclc/corc/index.htm

Desire information gateways handbook. Última actualización: 26 de abril de 2000. Desire Consortium, c1999-2000. Consultado en: 26-07-00. http://www.desire.org/handbook.

Desire project: development of an European service for information on research and education. Desire Consortium, c1998-2000. Consultado en: 1509-00.

http://www.desire.org

Dolin, R.; Agrawal, D.; El Abbadi, A; Pearlman, J. "Using automated classification for summarizing and selecting heterogeneous information sources. En: D-Lib magazine, 1998, enero. Consultado en: 18-06-00. http://www.dlib.org/dlib/january98/dolin/01dolin.html

Draft standard Z39.85-200X: the Dublin core metadata element set. National information standards organization, c2000, modificado: julio de 2000. Consultado en: 24-07-00.

http://www.niso.org/Z3985.html

Dublin core metadata initiative. DCI, c2000. Consultado en: 20-07-00. http://purl.org/DC/

ELib: the electronic libraries programme. ELib, última revisión: 6 de julio de 2000. Consultado en: 24-07-00.

http://www.ukoln.ac.uk/services/elib/

Estivill, Assumpció. "El processament dels recursos electrònics". En: BiD: textos universitaris de biblioteconomia i documentació, 1998, junio, n. 1. Consultado en: 15-07-00.

http://www.ub.es/biblio/bid/01estiv1.htm

García Testal, Cristina. "Corc: hacia la biblioteca digital". En: El profesional de la información, 2000, v. 9, n. 4, abril, pp. 17-20.

Gorman, Michael. "The future of cataloguing and cataloguers". En: Ifla general conference, 1997. Booklet 4, Division of bibliographic control, pp. 37-38.
http://www.ifla.org/IV/ifla63/63gorm.htm.

Guide for written collection policy statements. Subcommittee to revise the guide for written collection policy statements, Administration of collection development committee, Collection management and development section, Association for Library Collections \& Technical Services. Joanne S. Anderson, editor. 2nd. ed. Chicago; London: American Library Association, 1996

Guide to review of library collections: preservation, storage, and withdrawal. Subcommittee on review of collections, Collection management and development committee, Resources section, Association for Library Collections \& Technical Services. Leonore Clark, editor. Chicago; London: American Library Association, 1991, pp. 23.

Hickey, Thomas B.; Childress, Eric; Watson, Bradley C. "The genesis and development of Corc as an Oclc office of research project". En: Oclc newsletter, 1999, mayo-junio, n. 239. Consultado en: 02-01-00.

http://www.oclc.org/oclc/new/n239/feature/02feature.htm

Hirons, Jean. Revising Aacr2 to accomodate seriality. Prepared by Jean Hirons and members of the Conser Aacr review task force; submitted to the Joint steering committee for revision of Aacr. Febrero de 2000. Consultado en: 25-08-00

http://www.nlc-bnc.ca/jsc/ch12.pdf

Hofman, Paul; Worsfold, Emma. Selection criteria for quality controlled information gateways. Última actualización: 17 de mayo de 1999. Desire-Re 1004. Consulta: 22-07-00.

http://www.ukoln.ac.uk/metadata/desire/quality/

IMesh: International collaboration on internet subject gateways. UK office for library and information networking, University of Bath, 3 de septiembre de 1999, última actualización: 20 de junio de 2000. Consultado en: 25-08-00

http://www.imesh.org/

The IMesh toolkit: an architecture and toolkit for distributed subject gateways. 1999, enero. Consultado en: 23-07-00.

http://www.imesh.org/toolkit/proposal/proposal.pdf

InterCAT project. Oclc. Consultado en: 22-07-00.

http://www.oclc.org/oclc/research/projects/intercat.htm

\section{Próximos números monográficos}

\section{Diciembre $2000 \quad$ Información en ciencias de la salud}

Marzo 200I Hosts y productores de bases de datos

Mayo 200I Información para la inteligencia competitiva

Los interesados pueden remitir notas, artículos, propuestas, publicidad, comentarios, etc., sobre estos temas a:

epi@sarenet.es 
Isaac network. Internet scout project, c2000. Consultado en: 23-07-00. http://scout.es.wisc.edu/research

Joint steering committee for revision of Anglo-American cataloguing rules. Consultado en: 25-07-00.

http://www.nlc-bnc.ca/jsc/

Kirriemuir, John. A brief survey of quality resource discovery systems. Version 2 (final). c1999, última modificación: 18 de enero de 2000. Consultado en: 25-07-00.

http://www.rdn.ac.uk/publications/studies/survey/

Kirriemuir, John, et al. "Cross-searching subject gateways". En: D-Lib magazine, 1998, enero. Consultado en: 23-07-00.

http://www.dlib.org/dlib/january98/01kirriemuir.html

Knudson, Frances L., et al. "Creating electronic journal web pages from opac records". En: Issues in science and technology librarianship, 1997, n. 15. Consultado en: 17-07-00.

http://www.library.ucsb.edu/istl/97-summer/article2.html

Koch, Traugott. "Quality-controlled subject gateways: definitions, typologies, empirical overview". En: Manuscript of the article published in the subject gateways special issue of online information review, 2000, febrero, v. 24:1. Consultado en: 15-09-00.

http://www.lub.lu.se/ traugott/OIR-SBIG.txt

Lim, Edward. "Pasarelas temáticas del sudeste asiático: análisis de sus métodos de clasificación”. En: Ifla council and general conference, 1999. Consultado en: 16-07-00.

http://ifla.inist.fr/IV/ifla65/papers/011-117s.htm

Los Alamos National Laboratory. Electronic journals. Lanl, c1998, 15 de enero de 1999. Consultado en: 10-07-00.

http://lib-www.lanl.gov/cgi-bin/ejrnlsrch.cgi

McKiernan, Gerry. Beyond bookmarks: schemes for organizing the web. Iowa State University Library, 2000, enero. Consultado en: 24-07-00. http://www.public.iastate.edu/ CYBERSTACKS/CTW.htm

Merlo, José A.; Gracia, Juan; Zapico, Felipe; Rodríguez Gairín, Josep M. "Darwin: una propuesta de organización y evaluación del conocimiento accesible en línea”. En: La representación y la organización del conocimiento en sus distintas perspectivas: su influencia en la recuperación de la información: actas del IV Congreso Isko-España, 1999, pp. 87-94.

Meta matters. National Library of Canada, c1999. Consultado en: 24-07-00. http://www.nla.gov.au/meta/

NetFirst: Oclc, c1995-2000. Accesible en www a los usuarios del servicio comercial FirstSearch de Oclc.

The nordic metadata projects. Última actualización: 21 de febrero de 2000. Consultado en: 24-07-00.

http://linnea.helsinki.fi/metal

Parent, Ingrid. "An update of the Isbd(S) working group”. En: Ifla council and general conference, 1999, pp. 143-169. Consultado en: 13-07-00. http://www.ifla.org/IV/ifla65/papers/143-169e.htm

Peset, M. Fernanda; Albiñana, Ricardo; Morales, Silvia. "Internet invisible: un recurso terciario en la Red". En: El profesional de la información, 2000, julio-agosto, v. 9, n. 7-8, pp. 19-22

Porter, G. Margaret; Bayard, Laura. "Including web sites in the online catalog: implications for cataloging, collection development, and access". En: The journal of academic librarianship, 1999, septiembre, v. 25, n. 5, pp. 390-394.
RDN: Resource discovery network. c1999-200, última actualización: 14 de julio de 2000. Consultado en: 26-07-00.

http://www.rdn.ac.uk/

Renardus. Última actualización: 1 de septiembre de 2000. Consultado en: 13-09-00.

http://www.renardus.org

Roads cataloguing guidelines. Rev. version (v. 1.0) by Michael Day, UK Office for library and information networking (Ukoln), University of Bath. Última modificación: 16 de junio de 1999. Consultado en: 23-07-00.

http://www.ukoln.ac.uk/metadata/roads/cataloguing/

Roszkowski, M; Lukas, C. "A distributed architecture for resource discovery using metadata". En: D-Lib magazine, 1998, junio. Consultado en: 2207-00.

http://www.dlib.org/dlib/june98/scout/06roszkowsli.html

Serrano, Jordi, et al. "DinaWEB: l'organització de recursos accessibles en línia a la biblioteca virtual de la Universitat Oberta de Catalunya”. En: VII jornades catalanes de documentació, 1999. Les biblioteques i els centres de documentació al segle XXI. Barcelona: Cobdc, 1999, pp. 445-453.

Subject gateways. Desire Consortium, c1998-2000, Última modificación: 04 de octubre de 1999. Consultado en: 25-07-00.

http://www.desire.org/html/subjectgateways/subjectgateways.html

Taylor, Arlene G.; Clemson, Patrice. "Access to networked documents: catalogs? search engines? both?". En: Oclc internet cataloging colloquium, 1996. Consultado en: 13-12-99.

http://www.oclc.org/oclc/man/colloq/toc.htm

University of California, San Diego. Libraries. Electronic journals, newsletters, etc. General browsing. Última modificación: 20 de octubre de 1999. Consultado en: 25-07-00.

http://gort.ucsd.edu/ejourn/index.html

University of Rochester. River Campus Libraries. Selection guidelines for free internet resources. Drafted by Electronic resources team, 1997, agosto. Consultado en: 23-07-00.

http://www.lib.rochester.edu/cat/select.htm

Vizine-Goetz, Diane. "Using library classification schemes for internet resources". En: Oclc, internet cataloging project colloquium, 1996. Consultado en: 23-07-00.

http://www.oclc.org/oclc/man/colloq/v-g.htm

Woodward, Jeannette. "Cataloging and classifying information resources on the internet". En: Annual review of information science and technology, 1996, v. 31, pp. 189-220.

Weber, Mary Beth. "Factors to be considered in the selection and cataloging of internet resources”. En: Library hi-tech, 1999, v. 17, n. 3, pp. 298303.

Worsfold, Emma; Hiom, Debra. "The Desire project: promoting and facilitating web usage among Europe's research community". Pre-print de un artículo que se publicará en New review of information networking. Consultado: 27-07-00.

http://www.desire.org/html/aboutus/background/nrin/nrin.html

Yale University. Library. Criteria for cataloging selected remote access electronic resources. Consultado en: 23-08-00.

http://www.library.yale.edu/cataloging/netinfolecatselect.html

Assumpció Estivill, Ernest Abadal. estivill@fbd.ub.es

abadal@fbd.ub.es 